Symmetries, Differential Equations and Applications (SDEA-II)

International Journal of Modern Physics: Conference Series

Vol. 38 (2015) 1560073 (16 pages)

(C) The Authors

DOI: $10.1142 / \mathrm{S} 2010194515600733$

\title{
A review on symmetries for certain Aedes aegypti models
}

\author{
Igor Leite Freire \\ Centro de Matemática, Computação e Cognição \\ Universidade Federal do $A B C$ - UFABC \\ Rua Santa Adélia, 166, Bairro Bangu, 09.210-170 \\ Santo André, SP - Brasil \\ igor.freire@ufabc.edu.br; igor.leite.freire@gmail.com \\ Mariano Torrisi \\ Dipartimento di Matematica e Informatica, \\ Università Degli Studi di Catania, \\ Viale Andrea Doria, 6, 95125 Catania, Italia \\ torrisi@dmi.unict.it; m.torrisi12@gmail.com
}

Published 30 April 2015

\begin{abstract}
We summarize our results related with mathematical modeling of Aedes aegypti and its Lie symmetries. Moreover, some explicit, group-invariant solutions are also shown. Weak equivalence transformations of more general reaction diffusion systems are also considered. New classes of solutions are obtained.
\end{abstract}

Keywords: Aedes aegypti mosquitoes; reaction-diffusion equation; model for population dynamics; equivalence transformations; symmetries; invariant solutions.

\section{Introduction}

In this paper we revisit our previous results ${ }^{1-4}$ using group methods for mathematical modeling of Aedes aegypti mosquitoes. This species is the main vector of dengue, a disease characterized, among other symptoms, by high fever and severe pain in the body, which can be fatal in many cases.

Such a disease mainly occurs in countries located in the tropical zone of the globe, particularly those with a hot and humid climate. Although for years it was essentially restricted to the mentioned zones, it may be possible that in the next decades places and countries that until now have never been affected by this plague will be as a result of global warming. Most interested in the investigation about dengue and its vector are those who are currently affected. Climate change around

This is an Open Access article published by World Scientific Publishing Company. It is distributed under the terms of the Creative Commons Attribution 3.0 (CC-BY) License. Further distribution of this work is permitted, provided the original work is properly cited. 
the world can lead to modifications of the weather and such modifications are sufficient to attract the attention of those living in places that in the next decades can develop hot and humid weather becoming eventually fruitful places for the reproduction of Aedes aegypty and, consequently, be potentially affected by the dissemination of dengue.

Our research about Aedes aegypti was first concerned with the dispersal dynamics of the mosquito. We introduced as a new mathematical model, a partial differential equation system and carried out a complete group classification with respect to some constitutive parameters characterizing the model. ${ }^{1,2}$ Then we investigated the nonlinear case admitting the largest symmetry Lie algebra. ${ }^{3}$ From the biological point of view, the last one corresponds to an infestation in a place where wind currents can be neglected.

Motivated by our previous results, ${ }^{1-3}$ we considered a wide class of nonlinear reaction-diffusion systems that enclose our models. ${ }^{4}$ Those classes possessed three arbitrary constitutive functions, some of them depending on more than one variable. Therefore the classical Lie group analysis is not appropriate for an investigation concerned with invariance properties. ${ }^{5,6}$ For this reason we looked at such classes from the point of view of equivalence transformations. ${ }^{8-10}$

The plan of the paper is as the follows: in Sec. 2 we recall some models for Aedes aegypti, following those that inspired us to introduce our systems considered. ${ }^{1,2}$ Next, in Sec. 3, we show the Lie point symmetry generators found in. ${ }^{1,2}$ From these Lie point symmetry generators, we get some explicit solutions for some of the models obtained during the group classification. In particular we study the nonlinear case, which biologically corresponds to an infection in region with weak wind currents. ${ }^{3}$ Looking for further generalizations of our models, we consider weak equivalence transformations ${ }^{4}$ in Sec. 5. Since we are revisiting our results, and in some sense, summarizing them, in some parts the presentation might closely follow the previous works. ${ }^{1-4}$

\section{New Models}

Although the Aedes aegypti diffuses dengue among humans, we consider only mosquitoes populations. ${ }^{1-3}$

The first PDE model of the Aedes aegypti population was introduced by Tahahashi et al. ${ }^{11}$ In that model the mosquito population was divided into two subpopulations: the winged $u$ and the aquatic $v$. Their corresponding balance equations in an adimensional form are written as the following class of nonlinear reaction diffusion systems

$$
\left\{\begin{array}{l}
u_{t}=u_{x x}-\nu u_{x}+\frac{\gamma}{k} v(1-u)-\mu_{1} u, \\
v_{t}=k(1-v) u-\left(\mu_{2}+\gamma\right) v
\end{array}\right.
$$

where $k, \gamma, \mu_{1}, \mu_{2}$ are parameters depending on the environment and $\nu$ is related to the wind flux, which is responsible for the advection motion of the winged 
population; $k$ being the ratio between the carrying capacities related to the amount of findable nutrients, $k_{1}$, and the occupation of the available breeders $k_{2}$; the specific rate of maturation of the aquatic form into winged female mosquitoes $\gamma ; \mu_{1}$ and $\mu_{2}$ are the mortality of the winged population and the mortality of aquatic population, respectively. Apart from $\nu$, all of the remaining parameters are strictly positive. Further details are given in Takahashi et. al.. ${ }^{11}$ Additionally, the reader interested in the dimensional version of system (1) is guided to that reference, where system (1) is deduced point by point from its dimensionalised partner.

Generalisations of the model (1) can be obtained by considering nonlinear diffusion and nonlinear wind advection. Then, in this case we can write

$$
\left\{\begin{array}{l}
u_{t}=\left(u^{p} u_{x}\right)_{x}-2 \nu u^{q} u_{x}+\frac{\gamma}{k} v(1-u)-\mu_{1} u \\
v_{t}=k(1-v) u-\left(\mu_{2}+\gamma\right) v
\end{array}\right.
$$

where $p, q \in \mathbb{R}$.

We can consider the interaction between both winged and aquatic populations to be weak, since they are located in two different environments. In this way, the logistic terms $\frac{\gamma}{k} v(1-u)$ and $k(1-v) u$ could be replaced. We removed those terms and based on the modeling of Proteus Mirabilis ${ }^{12}$ they were substituted, respectively, by $\frac{\gamma}{k}(v+u)$ and $k(u+v)$, that is, we introduced the system

$$
\left\{\begin{array}{l}
u_{t}=\left(u^{p} u_{x}\right)_{x}-2 \nu u^{q} u_{x}+\frac{\gamma}{k} v+\left(\frac{\gamma}{k}-\mu_{1}\right) u \\
v_{t}=k u+\left(k-\mu_{2}-\gamma\right) v
\end{array}\right.
$$

System (3) can be considered as a Malthusian model ${ }^{13}$ (that is, without saturation of mosquitoes's growth). Therefore, from the biomathematical point of view, it is well suited for short time scales, that biologically may correspond to a fast infestation due to consecutive rainy days.

For further developments in this paper we remark that all of the systems shown in this section belong to the following class of nonlinear advection-reaction-diffusion equations

$$
\left\{\begin{array}{l}
u_{t}=\left(f(u) u_{x}\right)_{x}+g\left(u, v, u_{x}\right) \\
v_{t}=h(u, v)
\end{array}\right.
$$

Such a system can describe the dynamics of two interacting population densities, $u$ and $v$. The first balance equation takes into account some advection effects $u_{x}$, together with the diffusion of the winged population $u$ and the reaction effects. In our cases the advection effects are due to external stimuli of wind effects on the individuals of the population $u$. In the balance equation for density $v$ only the socalled reaction terms are taken into account. This equation is concerned with the aquatic population. 


\section{Symmetries}

In our previous researches symmetries of Aedes aegypti models ${ }^{1,3}$, we focused our attention in the system (3) because the system (1) only admits translations in space and time. In order to show some results obtained in ${ }^{1}$ we recall here a few elements about symmetries.

Definition 3.1. A symmetry for the system (3) is a non-degenerate point transformation of independent and depend variables

$$
T:=\left\{\begin{array}{l}
x^{*}=x^{*}(x, t, u, v), \\
t^{*}=t^{*}(x, t, u, v), \\
u^{*}=u^{*}(x, t, u, v), \\
v^{*}=v^{*}(x, t, u, v),
\end{array}\right.
$$

which leaves the system invariant.

Definition 3.2. A continuous one-parameter (local) Lie group of transformations is a family $G$ of transformations

$$
T_{\epsilon}:=\left\{\begin{array}{l}
x^{*}=x^{*}(x, t, u, v, \epsilon), \\
t^{*}=t^{*}(x, t, u, v, \epsilon), \\
u^{*}=u^{*}(x, t, u, v, \epsilon), \\
v^{*}=v^{*}(x, t, u, v, \epsilon),
\end{array}\right.
$$

which is locally a $C^{\infty}$-diffeomorphism in a subset $S \subseteq \mathbb{R}^{2+2}$ with coordinates $(x, t, u, v)$, depending analytically on the parameter $\varepsilon$ in a neighborhood $D \subseteq \mathbb{R}$ of $\varepsilon=0$ and reduces to the identity transformation when $\varepsilon=0$.

The linear form of $(6)$

$$
\begin{aligned}
& x^{*}=x+\epsilon \xi^{1}(x, t, u, v)+O\left(\epsilon^{2}\right), \\
& t^{*}=t+\epsilon \xi^{2}(x, t, u, v)+O\left(\epsilon^{2}\right), \\
& u^{*}=u+\epsilon \eta^{1}(x, t, u, v)+O\left(\epsilon^{2}\right), \\
& v^{*}=v+\epsilon \eta^{2}(x, t, u, v)+O\left(\epsilon^{2}\right),
\end{aligned}
$$

where

$$
\begin{gathered}
\xi^{1}(x, t, u, v):=\left.\frac{\partial x^{*}}{\partial \epsilon}\right|_{\varepsilon=0}, \quad \xi^{2}(x, t, u, v):=\left.\frac{\partial t^{*}}{\partial \epsilon}\right|_{\varepsilon=0}, \\
\eta^{1}(x, t, u, v):=\left.\frac{\partial u^{*}}{\partial \epsilon}\right|_{\varepsilon=0}, \quad \eta^{2}(x, t, u, v):=\left.\frac{\partial v^{*}}{\partial \epsilon}\right|_{\varepsilon=0},
\end{gathered}
$$

allow us to introduce the vector field

$$
X=\xi^{1}(x, t, u, v) \frac{\partial}{\partial x}+\xi^{2}(x, t, u, v) \frac{\partial}{\partial t}+\eta^{1}(x, t, u, v) \frac{\partial}{\partial u}+\eta^{2}(x, t, u, v) \frac{\partial}{\partial v} .
$$

This operator is usually called an infinitesimal generator of the transformation or infinitesimal generator of the Lie point symmetry. Then, given a transformation (7), it is possible to obtain the corresponding generator (8). Vice-versa, given a generator 
of the type (8), it is possible to obtain its transformation using the exponential map, that is, the transformation is given by $(x, t, u, v) \mapsto\left(e^{\epsilon X} x, e^{\epsilon X} y, e^{\epsilon X} u, e^{\epsilon X} v\right)$. The set of all infinitesimal generators leaving invariant a given equation endowed with the commutator $[X, Y]=X Y-Y X$, necessarily form a Lie algebra.

With respect to $(3)$, its Lie point symmetry generators were obtained ${ }^{1}$ by using the Lie infinitesimal criterion and the system (3) was completely classified with respect to the constitutive parameters $p, q, \nu$. The result obtained there are summarized in the following Table 1:

\begin{tabular}{|c|c|c|c|}
\hline$\nu$ & $p$ & $q$ & Generators \\
\hline$\forall$ & $\forall$ & $\forall$ & $\partial_{x}, \partial_{t}$ \\
\hline 0 & 0 & 0 & $\partial_{x}, \partial_{t}, u \partial_{u}+v \partial_{v}, B(x, t) \partial_{u}+B(x, t) \partial_{v}$ \\
\hline 0 & $-\frac{4}{3}$ & $\forall$ & $\partial_{x}, \partial_{t}, x^{2} \partial_{x}-3 x u \partial_{u}-3 x v \partial_{v}, 2 x \partial_{x}-3 u \partial_{u}-3 v \partial_{v}$ \\
\hline 0 & $\neq-\frac{4}{3}, 0$ & $\forall$ & $\partial_{x}, \partial_{t}, p x \partial_{x}+2 u \partial_{u}+2 v \partial_{v}$ \\
\hline$\neq 0$ & $2 q$ & $\neq 0$ & $\partial_{x}, \partial_{t}, q x \partial_{x}+u \partial_{u}+v \partial_{v}$ \\
\hline
\end{tabular}

The functions $(B, \tilde{B})$ appearing in the second row of the Table 1 are solutions of the following linear system

$$
\left\{\begin{array}{l}
B_{t}=B_{x x}+\frac{\gamma}{k} \tilde{B}+\left(\frac{\gamma}{k}-\mu_{1}\right) B \\
\tilde{B}_{t}=k B+\left(k-\mu_{2}-\gamma\right) \tilde{B}
\end{array}\right.
$$

Remark 3.1. Although system (3) has the parameters $\gamma, \mu_{1}, \mu_{2}, k$, as well as $p, q$ and $\nu$, only the last three affect the group classification in the nonlinear cases. When the nonlinearities are removed, the remaining parameters influence the symmetry group in the sense that the solution of (9) depend on the parameters $\gamma, \mu_{1}, \mu_{2}, k$ and, consequently, the generator $B(x, t) \partial_{u}+\tilde{B}(x, t) \partial_{v}$ has some dependence on these constitutive parameters.

\section{Invariant Solutions}

In this section we give a short review of some reductions of the system (3) derived in references. ${ }^{1,3}$

\subsection{Case $\nu \neq 0$}

In this case we have an advection due to wind currents, acting on the winged density population $u$.

\subsubsection{The case $p=2 q \neq 0$}

In this subcase, the symmetries are translations in space and time, and a dilation $(x, t, u, v) \mapsto\left(\lambda^{q} x, t, \lambda u, \lambda v\right), \lambda>0$. Then, the linear combination of the Lie point symmetry generators is given by

$$
X=c_{3} \partial_{t}+\left(q x+c_{2}\right) \partial_{x}+u \partial_{u}+v \partial_{v} .
$$




\section{L. Freire \&3 M. Torrisi}

The invariant surface conditions imply

$$
\sigma=\frac{e^{\frac{q}{c_{3}} t}}{q x+c_{2}}, \quad u=e^{\frac{t}{c_{3}}} U(\sigma), \quad v=e^{\frac{t}{c_{3}}} V(\sigma) .
$$

Then system (3), after having puting $p=2 q$, gives the reduced ODE system:

$$
\begin{aligned}
\frac{1}{c_{3}}\left(U+q \sigma U^{\prime}\right)= & 2 q^{3} \sigma^{4} U^{2 q-1}\left(U^{\prime}\right)^{2}+2 q^{2} \sigma^{3} U^{2 q} U^{\prime}+q^{2} \sigma^{4} U^{2 q} U^{\prime \prime} \\
& +2 \nu q \sigma^{2} U^{q} U^{\prime}+\frac{\gamma}{k} V+\left(\frac{\gamma}{k}-\mu_{1}\right) U, \\
\frac{1}{c_{3}}\left(V+q \sigma V^{\prime}\right)= & k U+\left(k-\mu_{2}-\gamma\right) V .
\end{aligned}
$$

In order to bring this system in an autonomous form we observe that the system (11) is invariant under the following associated ${ }^{14}$ dilation transformation group

$$
\sigma=a^{-1} \sigma^{\star}, \quad U=a^{\frac{1}{q}} U^{\star}, \quad V=a^{\frac{1}{q}} V^{\star} .
$$

We can do the following change of variables, ${ }^{15-18}$

$$
\eta=\ln \sigma, \quad V_{1}(\eta)=\frac{U}{\sigma^{-\frac{1}{q}}}, \quad V_{2}(\eta)=\frac{V}{\sigma^{-\frac{1}{q}}} .
$$

Putting $V_{i, \eta}:=\frac{d V_{i}}{d \eta}$ it is possible to rewrite system (11) in the following autonomous form

$$
\begin{aligned}
V_{1, \eta}= & 2 q^{3} V_{1}^{2 q-1}\left(V_{1, \eta}-\frac{1}{q} V_{1}\right)^{2}+2 q^{2} V_{1}^{2 q}\left(V_{1, \eta}-\frac{1}{q} V_{1}\right) \\
& +V_{1}^{2 q} q^{2}\left[V_{1, \eta \eta}-\frac{1}{q} V_{1, \eta}-\left(\frac{1}{q}+1\right)\left(V_{1, \eta}-\frac{1}{q} V_{1}\right)\right] \\
& +2 q \nu V_{1}^{q}\left(V_{1, \eta}-\frac{1}{q} V_{1}\right)+\frac{\gamma}{k} V_{2}+\left(\frac{\gamma}{k}-\mu_{1}\right) V_{1}, \\
V_{2, \eta}= & k V_{1}+\left(k-\mu_{2}-\gamma\right) V_{2} .
\end{aligned}
$$

which can be written as a $3 \times 3$ dynamical system.

\subsubsection{The case $p \neq 2 q$}

In this subcase the only admitted symmetries are the temporal translations and the spatial translations. Therefore we can look for invariant solutions of the form $u=$ $U(\sigma)$ and $v=V(\sigma)$ where $\sigma=x-\omega t$ where $\omega$ is an arbitrary constant representing the velocity of wave propagation. In this case the reduced system assumes the following form:

$$
\begin{aligned}
& -\omega U^{\prime}=\left(U^{p} U^{\prime}\right)^{\prime}-2 \nu U^{q} U^{\prime}+\frac{\gamma}{k} V+\left(\frac{\gamma}{k}-\mu_{2}\right) U, \\
& -\omega V^{\prime}=k U+\left(k-\mu_{2}-\gamma\right) V .
\end{aligned}
$$

As in the previous system, we can rewrite (14) like a $3 \times 3$ first order system to perform a qualitative analysis. If we are interested in a formal solution, it is possible to verify its existence, at least for some special values of $p$ and $q$. 
For instance if we take $p=0$ and $q=1$ it is easy to verify that

$$
U=A_{1} \sigma+A_{0}, \quad V=B_{1} \sigma+B_{0},
$$

are solutions of (14) provided that $A_{1}, A_{0}, B_{1}, B_{0}$ are solutions of the following nonlinear algebraic system

$$
\begin{aligned}
& \omega A_{1}=2 \nu A_{0} A_{1}-\frac{\gamma}{k} B_{0}-\left(\frac{\gamma}{k}-\mu_{2}\right) A_{0}, \\
& 2 \nu A_{1}^{2}-\left(\frac{\gamma}{k}-\mu_{2}\right) A_{1}-\frac{\gamma}{k} B_{1}=0, \\
& -\omega B_{1}=k A_{0}+\left(k-\mu_{2}-\gamma\right) B_{0}, \\
& k A_{1}+\left(k-\mu_{2}-\gamma\right) B_{1}=0 .
\end{aligned}
$$

without requiring restrictions on the constitutive parameters.

\subsection{Case $\nu=0$}

This case corresponds to a situation where the wind currents can be neglected. For instance, it might correspond to an infestation in a small region, between builds, in which wind currents are very weak. Using Table 1 we can distinguish two possibilities

\subsection{1. $p=-4 / 3$}

In this subcase the system $(3)$ reads

$$
\left\{\begin{array}{l}
u_{t}=\left(u^{-\frac{4}{3}} u_{x}\right)_{x}+\frac{\gamma}{k} v+\left(\frac{\gamma}{k}-\mu_{1}\right) u \\
v_{t}=k u+\left(k-\mu_{2}-\gamma\right) v
\end{array}\right.
$$

whose Lie algebra, taking the results obtained in ${ }^{1}$ into account, is spanned by

$$
X_{1}=\partial_{x}, \quad X_{2}=\partial_{t}, \quad X_{p}=p x \partial_{x}+2 u \partial_{u}+2 v \partial_{v},
$$

with $p=-4 / 3$ in $X_{p}$, and by the additional generator

$$
X_{3}=x^{2} \partial_{x}-3 x u \partial_{u}-3 x v \partial_{v} .
$$

From this generator, we can look for an invariant solution of the form

$$
u(x, t)=\frac{A(t)}{x^{3}}, \quad v(x, t)=\frac{B(t)}{x^{3}} .
$$

Substituting these functions into (3) and taking into account that $\nu=0$ and $p=$ $-4 / 3$, we obtain the following linear system of ordinary differential equations

$$
\begin{aligned}
& A^{\prime}=\frac{\gamma}{k} B+\left(\frac{\gamma}{k}-\mu_{1}\right) A, \\
& B^{\prime}=k A+\left(k-\mu_{2}-\gamma\right) B,
\end{aligned}
$$

whose solutions are

$$
A(t)=a_{1} e^{-\frac{b t}{2}} \cosh \left(\frac{\sqrt{\Delta} t}{2}\right)+a_{2} e^{-\frac{b t}{2}} \sinh \left(\frac{\sqrt{\Delta} t}{2}\right),
$$




$$
\begin{aligned}
B(t)= & a_{1} \frac{e^{-\frac{b t}{2}}}{2}\left[\sqrt{\Delta} \sinh \left(\frac{\sqrt{\Delta} t}{2}\right)-b \cosh \left(\frac{\sqrt{\Delta} t}{2}\right)\right] \\
& +a_{2} \frac{e^{-\frac{b t}{2}}}{2}\left[\sqrt{\Delta} \cosh \left(\frac{\sqrt{\Delta} t}{2}\right)-b \sinh \left(\frac{\sqrt{\Delta} t}{2}\right)\right]
\end{aligned}
$$

where $a_{1}, a_{2}$ are arbitrary constants and

$$
\begin{gathered}
\Delta=4 \gamma+\left[\left(\mu_{1}-\frac{\gamma}{k}\right)+\left(k-\mu_{2}-\gamma\right)\right]^{2}>0, \\
b=\mu_{1}+\mu_{2}+\gamma-k-\frac{\gamma}{k}, c=-\gamma+\left(\frac{\gamma}{k}-\mu_{1}\right)\left(k-\mu_{2}-\gamma\right) .
\end{gathered}
$$

A particular solution is given by

$$
u(x, t)=e^{-\frac{b t}{2}} \frac{\cosh \left(\frac{\sqrt{\Delta} t}{2}\right)}{x^{3}}, \quad v(x, t)=\frac{e^{-\frac{b t}{2}}}{2 x^{3}}\left[\sqrt{\Delta} \sinh \left(\frac{\sqrt{\Delta} t}{2}\right)-b \cosh \left(\frac{\sqrt{\Delta} t}{2}\right)\right] .
$$

Recalling that $(x, t, u, v) \mapsto\left(x+x_{0}, t, u, v\right)$ and $(x, t, u, v) \mapsto\left(x, t+t_{0}, u, v\right)$ are symmetries, we can obtain a "new solution" from the previous one, given by

$$
\begin{aligned}
& \tilde{u}(x, t)=e^{-\frac{b\left(t+t_{0}\right)}{2}} \frac{\cosh \left(\frac{\sqrt{\Delta}\left(t+t_{0}\right)}{2}\right)}{\left(x+x_{0}\right)^{3}}, \\
& \tilde{v}(x, t)=\frac{e^{-\frac{b\left(t+t_{0}\right)}{2}}}{2\left(x+x_{0}\right)^{3}}\left[\sqrt{\Delta} \sinh \left(\frac{\sqrt{\Delta}\left(t+t_{0}\right)}{2}\right)-b \cosh \left(\frac{\sqrt{\Delta}\left(t+t_{0}\right)}{2}\right)\right] .
\end{aligned}
$$

A more general solution was obtained. ${ }^{3}$ Consider the linear combination

$$
X=\left(c_{1}+2 x c_{4}+c_{3} x^{2}\right) \partial_{x}+c_{2} \partial_{t}-\left(3 x u c_{3}+3 u c_{4}\right) \partial_{u}-\left(3 x v c_{3}+3 v c_{4}\right) \partial_{v}
$$

of the basis of the Lie symmetry algebra for the system (15). Then, by assuming $c_{3}=1$, from the invariant surface conditions, we get the following invariant solutions

$$
u=\frac{A(\phi)}{\left(x^{2}+2 c_{4} x+c_{1}\right)^{3 / 2}}, \quad v=\frac{B(\phi)}{\left(x^{2}+2 c_{4} x+c_{1}\right)^{3 / 2}},
$$

where

$$
\begin{aligned}
\phi & =t-c_{2} \int \frac{d x}{x^{2}+2 c_{4} x+c_{1}} \\
& = \begin{cases}t+\frac{c_{2}}{x+c_{4}}, & \text { if } c_{4}^{2}-c_{1}=0, \\
t-\frac{c_{2}}{\sqrt{c_{1}-c_{4}^{2}}} \arctan \left(\frac{x+c_{4}}{\sqrt{c_{1}-c_{4}^{2}}}\right), & \text { if } c_{4}^{2}-c_{1}<0, \\
t-\frac{c_{2}}{2 \sqrt{c_{4}^{2}-c_{1}}} \ln \left|\frac{x+c_{4}-\sqrt{c_{4}^{2}-c_{1}}}{x+c_{4}+\sqrt{c_{4}^{2}-c_{1}}}\right|, & \text { if } c_{4}^{2}-c_{1}>0 .\end{cases}
\end{aligned}
$$


If we neglect time translations, that is, $c_{2} \rightarrow 0$ in (21) and, consequently, in (23), the reduced system becomes

$$
\begin{aligned}
& A^{\prime}=-3 A^{-\frac{1}{3}}\left(c_{1}-c_{4}^{2}\right)+\frac{\gamma}{k} B+\left(\frac{\gamma}{k}-\mu_{1}\right) A, \\
& B^{\prime}=k A+\left(k-\mu_{2}-\gamma\right) B
\end{aligned}
$$

in the unknown functions $A(t)$ and $B(t)$.

The solutions of system (24) are not easy to obtain due to the nonlinearity in the first equation. However, when $c_{1}=c_{4}^{2}$, it can be reduced to a single linear ordinary differential equation. In fact it is the system (18). For further details, see. ${ }^{3}$

\subsection{2. $\forall p \notin\{-4 / 3,0\}$}

In this subcase the system (3) reads

$$
\left\{\begin{array}{l}
u_{t}=\left(u^{p} u_{x}\right)_{x}+\frac{\gamma}{k} v+\left(\frac{\gamma}{k}-\mu_{1}\right) u \\
v_{t}=k u+\left(k-\mu_{2}-\gamma\right) v
\end{array}\right.
$$

Its 3-dimensional Lie algebra is spanned by (16). We consider the following general infinitesimal operator

$$
X=p c_{1} X_{1}+c_{2} X_{2}+X_{p}
$$

where $c_{1}$ and $c_{2}$ are arbitrary real constants.

By applying the invariant surface conditions we conclude that the invariant solutions, with respect to (26), have the form:

$$
u(x, t)=A(\sigma)\left(x+c_{1}\right)^{\frac{2}{p}}, \quad v(x, t)=B(\sigma)\left(x+c_{1}\right)^{\frac{2}{p}},
$$

where

$$
\sigma=\frac{e^{t}}{\left(c_{1}+x\right)^{\frac{c_{2}}{p}}}
$$

and the functions $A(\sigma)$ and $B(\sigma)$ satisfy the following general reduced system of ODEs

$$
\left\{\begin{aligned}
A^{\prime} \sigma= & \left(2 A-A^{\prime} \sigma\right)^{2}+\frac{\gamma}{k} B+\left(\frac{\gamma}{k}-\mu_{1}\right) A \\
& +A^{p}\left[\left(\frac{4}{p^{2}}-\frac{2}{p}\right) A+A^{\prime} \frac{\sigma}{p}\left(1-c_{2}\right)\left(1+\frac{2}{p}\right)+\frac{c_{2} A^{\prime \prime} \sigma^{2}}{p}\right] \\
B^{\prime} \sigma= & k A+\left(k-\mu_{2}-\gamma\right) B .
\end{aligned}\right.
$$

As in the previous case, we neglect translations in time. Then (27) can be written as

$$
\left\{\begin{array}{l}
A^{\prime}(t)=\frac{4+2 p}{p^{2}} A(t)^{p+1}+\frac{\gamma}{k} B(t)+\left(\frac{\gamma}{k}-\mu_{1}\right) A(t), \\
B^{\prime}(t)=k A(t)+\left(k-\mu_{2}-\gamma\right) B(t) .
\end{array}\right.
$$

It is easy to ascertain by simple calculations that it is possible to reduce the search for solutions of (28) to the search for solutions of the following second order 
ODE:

$$
\begin{aligned}
A^{\prime \prime}(t) & -\left(\frac{(p+1)(4+2 p)}{p^{2}} A^{p}(t)+\left(\frac{\gamma}{k}-\mu_{1}+k-\mu_{2}-\gamma\right)\right) A^{\prime}(t) \\
& +\left(\left(k-\mu_{2}-\gamma\right) \frac{4+2 p}{p^{2}} A(t)^{p}-\gamma+\left(k-\mu_{2}-\gamma\right)\left(\frac{\gamma}{k}-\mu_{1}\right)\right) A(t)=0,
\end{aligned}
$$

that is a family of Lienard equations. After having put

$$
a=\frac{(p+1)(4+2 p)}{p^{2}}, \quad d=\left(k-\mu_{2}-\gamma\right) \frac{4+2 p}{p^{2}}
$$

we are able to write $(29)$ as

$$
A^{\prime \prime}(t)-\left(a A^{p}-b\right) A^{\prime}+\left(d A^{p}+c\right) A=0,
$$

where $b$ and $c$ are given by (20). Some classes of solutions of this family were obtained. ${ }^{3}$

\section{Weak Equivalence Transformations and Symmetries for the System (4)}

We consider, now, the following class of nonlinear advection-reaction-diffusion equations

$$
\left\{\begin{array}{l}
u_{t}=\left(f(u) u_{x}\right)_{x}+g\left(u, v, u_{x}\right), \quad g_{u_{x}} \neq 0, \\
v_{t}=h(u, v)
\end{array}\right.
$$

from the point of view of weak equivalence transformations. ${ }^{4}$ After reviewing this work we apply our results for other models that are potentially interesting from the biomathematical point of view.

\subsection{Equivalence transformations}

Following Ibragimov et al., ${ }^{7}$ we consider an equivalence transformation a non degenerated change of independent and dependent variables $t, x, u, v$ into $\hat{t}, \hat{x}, \hat{u}, \hat{v}$, such that

$$
x=x(\hat{x}, \hat{t}, \hat{u}, \hat{v}), \quad t=t(\hat{x}, \hat{t}, \hat{u}, \hat{v}), \quad u=u(\hat{x}, \hat{t}, \hat{u}, \hat{v}), \quad v=v(\hat{x}, \hat{t}, \hat{u}, \hat{v}),
$$

that maps a system of the class (32) in another one preserving the differential structure but, in general, with $\hat{f}(\hat{u}) \neq f(u), \hat{g}\left(\hat{u}, \hat{v}, \hat{u}_{\hat{x}}\right) \neq g\left(u, v, u_{x}\right), \hat{h}(\hat{u}, \hat{v}) \neq$ $h(u, v)$. In the special case in which $\hat{f}(\hat{u})=f(u), \hat{g}\left(\hat{u}, \hat{v}, \hat{u}_{\hat{x}}\right)=g\left(u, v, u_{x}\right)$ and $h(\hat{u}, \hat{v})=h(u, v)$ an equivalence transformation is a point symmetry.

Following Ovsiannikov, ${ }^{5}$ equivalence transformations of systems of the type (32) can be considered as transformations acting on the basic augmented space $A \equiv$ $\left\{t, x, u, v, u_{x}, f, g, h\right\}$. 
Then we can consider a family of one-parameter equivalence transformations as a group of transformations, acting on the basic augmented space $A$, of the type

$$
\left\{\begin{array}{l}
x=x(\hat{x}, \hat{t}, \hat{u}, \hat{v}, \varepsilon) \quad t=t(\hat{x}, \hat{t}, \hat{u}, \hat{v}, \varepsilon), \quad u=u(\hat{x}, \hat{t}, \hat{u}, \hat{v}, \varepsilon), \quad v=v(\hat{x}, \hat{t}, \hat{u}, \hat{v}, \varepsilon), \\
f=f(\hat{x}, \hat{t}, \hat{u}, \hat{v}, \varepsilon), \quad g=g\left(\hat{x}, \hat{t}, \hat{u}, \hat{v}, \hat{u}_{x}, \varepsilon\right), \quad h=h(\hat{x}, \hat{t}, \hat{u}, \hat{v}, \varepsilon)
\end{array}\right.
$$

which is locally a $C^{\infty}$-diffeomorphism, depending analytically on the parameter $\varepsilon$ in a neighbourhood of $\varepsilon=0$ and reduces to the identity transformation for $\varepsilon=0$.

Following ${ }^{5-10,19,20}$ we consider the infinitesimal generator of the equivalence transformations of the system (32) that reads:

$$
Y=\xi^{1} \partial_{x}+\xi^{2} \partial_{t}+\eta^{1} \partial_{u}+\eta^{2} \partial_{v}+\mu^{1} \partial_{f}+\mu^{2} \partial_{g}+\mu^{3} \partial_{h}
$$

where all infinitesimal components $\xi^{1}, \xi^{2}, \eta^{1}, \eta^{2}$ and $\mu^{i}(i=1,2,3)$ are sought, at least in principle, depending on $x, t, u, v, u_{x}, f, g$ and $h$. In order to obtain the determining system, which allows us to get the infinitesimal coordinates $\xi^{i}, \eta^{i}$ and $\mu^{j}$ ( $i=1,2$ and $j=1,2,3$ ), we apply the Lie-Ovsiannikov's infinitesimal criterion. In this case, according to our results, ${ }^{4}$ the generator of weak (see, e.g ${ }^{9,10,12}$ ) equivalence transformations of (32) is given by the operator

$$
\begin{aligned}
Y= & \alpha(x) \partial_{x}+\beta(t) \partial_{t}+\delta(t, u) \partial_{u}+\lambda(x, t, v) \partial_{v}+\left(2 \alpha^{\prime}-\beta^{\prime}\right) f \partial_{f} \\
& +\left(\delta_{t}+\left(\delta_{u}-\beta_{t}\right) g+\left(\alpha^{\prime \prime}-\delta_{u u} u_{x}\right) f\right) \partial_{g}+\left(\left(\lambda_{v}-\beta^{\prime}\right) h+\lambda_{t}\right) \partial_{h}
\end{aligned}
$$

Here we recall that a weak equivalence transformation is still a transformation of the type (33) but it can map the arbitrary functions $f, g$, and $h$ into another functions that not only have different form but also have additional functional dependencies. Their generators are obtained by simplifying the Lie-Ovsiannikov algorithm. Further details concerned with the weak equivalence of system (32) and similar systems can be found in references. ${ }^{4,12}$

\subsection{Symmetries for the system (32)}

We recall the following theorem, proved by us. ${ }^{4}$ For related material see references. $^{21-25}$

Theorem 5.1. Let (36) be an infinitesimal equivalence generator for the systems (32), then the operator

$$
X=\alpha(x) \partial_{x}+\beta(t) \partial_{t}+\delta(t, u) \partial_{u}+\lambda(x, t, v) \partial_{v}
$$

which corresponds to the projection of $Y$ on the space $(x, t, u, v)$, is an infinitesimal symmetry generator of the system (32) if and only if the constitutive equations, specifying the forms of $f, h$ and $g$, are invariant with respect to $Y$. 


\section{L. Freire \&3 M. Torrisi}

For the system under consideration, in general, the constitutive equations whose invariance must be requested are:

$$
\left\{\begin{array}{l}
f=D(u), \\
g=G\left(u, v, u_{x}\right), \\
h=F(u, v) .
\end{array}\right.
$$

The invariance condition reads

$$
\left.Y(f-D(u))\right|_{38}=0,\left.\quad Y^{(1)}\left(g-G\left(u, v, u_{x}\right)\right)\right|_{38}=0,\left.\quad Y(h-F(u, v))\right|_{(38)}=0,
$$

where

$$
Y^{(1)}=Y+\zeta_{j}^{\alpha} \partial_{u_{j}^{\alpha}}+\omega_{a}^{A} \partial_{h_{a}^{A}}
$$

is the first extension of (36) and

$$
\zeta_{j}^{\alpha}=D_{j}^{e} \eta^{\alpha}-u_{k}^{\alpha} D_{j}^{e} \xi^{k}, \quad \zeta_{i j}^{\alpha}=D_{j}^{e} \zeta_{j}^{\alpha}-u_{i k}^{\alpha} D_{j}^{e} \xi^{k}, \quad \omega_{a}^{A}=\tilde{D}_{a}^{e} \mu^{A}-h_{b}^{A} \tilde{D}_{a}^{e} \nu^{b} .
$$

For further details, see. ${ }^{4,9,10,19}$

From the invariance condition (40), we obtain the following equations

$$
\mu^{1}-\eta^{1} D_{u}=0, \quad \mu^{2}-\zeta_{1}^{1} G_{u_{x}}-\eta^{1} G_{u}-\eta^{2} G_{v}=0, \quad \mu^{3}-\eta^{1} F_{u}-\eta^{2} F_{v}=0
$$

restricted to $(39)$.

Substituting

$$
\zeta_{1}^{1}=\left(\eta_{u^{1}}^{1}-\xi_{1}^{1}\right) u_{1}^{1}=\left(\delta_{u}-\alpha^{\prime}\right) u_{x}
$$

and taking into account (39) we can rewrite the equations (41) in the following form

$$
\begin{aligned}
& \left(2 \alpha^{\prime}-\beta^{\prime}\right) D(u)-\delta(t, u) D_{u}=0, \\
& \delta_{t}+\left(\delta_{u}-\beta\right) G-\left(\delta_{u u} u_{x}^{2}-\alpha^{\prime \prime} u_{x}\right) D-\left(\delta_{u}-\alpha^{\prime}\right) u_{x} G_{u_{x}} \\
& \quad-\delta G_{u}-\lambda(t, x, v) G_{v}=0, \\
& \left(\left(\lambda_{v}-\beta^{\prime}\right) F+\lambda_{t}\right)-\delta F_{u}-\lambda(t, x, v) F_{v}=0 .
\end{aligned}
$$

It is easy to see that the translation generators

$$
X_{0}=\partial_{t}, \quad X_{1}=\partial_{x}
$$

are Lie point symmetries of (32) for any functions $f(u), g\left(u, v, u_{x}\right)$ and $h(u, v)$. In particular, they are the only members of the principal Lie algebra $L_{\mathcal{P}}$ of the equivalence algebra. $\mathrm{In}^{4}$ we proved

Corollary 5.1. An equivalence operator for the system (32) belongs to Principal Lie Algebra $L_{\mathcal{P}}$ iff $\eta^{i}=0, \mu^{j}=0, i=1,2, j=1,2,3$. 


\subsection{Some extensions of the principal Lie algebra}

In order to show how to get some possible extensions of the Principal Algebra from (43)-(45) we assume that

$$
G=\rho u^{r} u_{x}+\Gamma_{1} u^{2}+\Gamma_{2} v
$$

where the parameters $\rho, r, \Gamma_{1}, \Gamma_{2}$ are constitutive parameters of the considered phenomena. Here, for the sake of simplicity, we assume $\rho, \Gamma_{1}, \Gamma_{2}, D_{u} \neq 0$. We would like to emphasize that in this section the form of the function $G$, as well as certain values of the parameters and the restrictions considered, although similar to some forms previously considered, were mainly chosen following a criterion of mathematical simplicity. From (43) we conclude that $\alpha=\alpha_{1} x+\alpha_{0}$, where $\alpha_{1}, \alpha_{0}$ are arbitrary constants. Then we can write (43) as

$$
\left(2 \alpha_{1}-\beta^{\prime}\right) D(u)-\delta(t, u) D_{u}=0
$$

while, after having taken (46) into account, (44) reads

$$
\begin{aligned}
\delta_{t}+ & \left(\delta_{u}-\beta_{t}\right)\left(\rho u^{r} u_{x}+\Gamma_{1} u^{2}+\Gamma_{2} v\right)-D \delta_{u u} u_{x}^{2}-\left(\delta_{u}-\alpha_{1}\right) \rho u^{r} u_{x} \\
& -\delta\left(r \rho u^{r-1} u_{x}+2 \Gamma_{1} u\right)-\lambda(t, x, v) \Gamma_{2}=0,
\end{aligned}
$$

which can itself be rewritten in the form

$\delta_{t}+\left(\delta_{u}-\beta^{\prime}\right)\left(\Gamma_{1} u^{2}+\Gamma_{2} v\right)-2 \Gamma_{1} \delta u-\lambda \Gamma_{2}+u_{x}\left[\left(\alpha^{\prime}-\beta^{\prime}\right) \rho u^{r}-r \delta \rho u^{r-1}\right]-D \delta_{u u} u_{x}^{2}=0$.

From the last equation, we can easily conclude that $\delta=\delta_{1}(t) u+\delta_{0}(t)$ from the coefficient of $u_{x}^{2}$. From the free term and the coefficient of $u_{x}$, we obtain

$$
\alpha=(1-r) a_{1} x+a_{0}, \quad \delta=-a_{1} u, \quad \beta=a_{1} t+a_{2}, \quad \lambda=-2 a_{1} v
$$

where $a_{0}, a_{1}$ and $a_{2}$ are arbitrary constants. Then, as a consequence of Theorem 1 , in addition to the translations in $x$ and $t$, we have the following dilation symmetry generator

$$
X=(1-r) x \frac{\partial}{\partial x}+t \frac{\partial}{\partial t}-u \frac{\partial}{\partial u}-2 v \frac{\partial}{\partial v}
$$

provided that $D$ and $F$ are solutions of the following differential equations

$$
u D_{u}=(2 r-1) D, \quad u F_{u}+2 v F_{v}=3 F
$$

\subsection{Reduction}

Let us take $r=1$. Then, a solution to (51) is given by $D=u$ and $F=a u^{3}+b v^{\frac{3}{2}}$, where $a$ and $b$ are constants. 
Using the generator (50), we have following system of ODEs:

$$
\frac{d x}{0}=\frac{d t}{t}=\frac{d u}{-u}=\frac{d v}{-2 v}
$$

which gives us

$$
u=\frac{f(x)}{t}, \quad v=\frac{g(x)}{t^{2}} .
$$

Substituting such function into the system (32), with $D=u, G=\rho u u_{x}+\Gamma_{1} u^{2}+$ $\Gamma_{2} v$ and $F=a u^{3}+b v^{\frac{3}{2}}$, we have

$$
\left\{\begin{array}{l}
-f=f f^{\prime \prime}+\rho f f^{\prime}+\Gamma_{1} f^{2}+\Gamma_{2} g, \\
-g=a f^{3}+b g^{\frac{3}{2}}
\end{array}\right.
$$

The second equation of (53) can be brought to a third degree algebraic equation for $g$ whose solution must be substituted in the first equation. Of course we only considered the positive root since $u$ and $v$ are densities and the negative value would guide us to a negative function, which does not have meaning in this case. Once solved the ODE for $f$ and $g$, we can easily obtain a solution for the system using $(52)$.

\section{Conclusions}

In this paper we revisited the main results of our previous papers ${ }^{1-4}$ concerned with the population dynamics of the Aedes aegypti, the main vector of dengue, obtained using the Lie group analysis techniques.

We observe that the theory of continuous symmetries enables us to obtain, from the system (3) five different models, corresponding to different cases with respect to the parameters $p, q$ and $\nu$, as it was summarized on Table 1 .

The parameters $p$ and $q$ are related, respectively, with nonlinearities in the diffusion and in the advection. On the other hand, the term $\nu$ corresponds to the wind influence on the Aedes aegypti models. It is interesting to observe that from the Lie point symmetry classification we must consider as special cases some that can have biological significance or meaning, such as the case $\nu=0$, which was extensively considered in. ${ }^{3}$

Inspired by models considered previously, in section 4 we show some applications of equivalence transformation techniques concerned with class (32) in order to get symmetries from new forms of the constitutive functions.

We have shown how it is easily possible, by applying the projection theorem (5.1), to get an idea of the form of the constitutive functions and determine the extensions of the principal Lie Algebra for the selected form of the constitutive functions. $^{4}$

\section{Acknowledgements}

The authors are grateful to FAPESP (project no. 2014/05024-8) for financial support. M.T. acknowledges the support from the GNFM (Gruppo Nazionale 
per Fisica-Matematica) of INdAM. I. L. Freire also thanks CNPq (process no. 308941/2013-6) for financial support.

\section{References}

1. I. L. Freire and M. Torrisi, Nonlin. Anal.: Real World Appl., 14, 1300-1307, (2013), http://dx.doi.org/10.1016/j.nonrwa.2012.09.018.

2. I. L. Freire and M. Torrisi, "On a Model for Aedes Aegypti" Numerical analysis and applied mathematics (ICNAAM 2012), Vols A and B, edited by: Simos, TE; Psihoyios, G; Tsitouras, C; et al. AIP Conference Proceedings 1479, American Institute of Physics, New York, 2012, pp. 1373-1376.

3. I. L. Freire and M. Torrisi, Commun. Nonlinear Sci. Numer. Simulat., 19, 872-879, (2014) http://dx.doi.org/10.1016/ j.cnsns.2013.08.006.

4. I. L. Freire and M. Torrisi, Abstr. Appl. Analysis, Art. Number: 546083, 2014, (2014), http://dx.doi.org/10.1155/2014/546083.

5. L. V. Ovsiannikov, Group Analysis of Differential Equations. Academic Press, New York, (1982).

6. N. H. Ibragimov, CRC Handbook of Lie Group Analysis of Differential Equations, Boca Raton, Florida: CRC Press, (1996).

7. N. H. Ibragimov, M. Torrisi and A. Valenti, Preliminary group classification of equation $v_{t t}=f\left(x, v_{x}\right) v_{x x}+g\left(x, v_{x}\right)$, J. Math. Phys., 32, 2988-2995, (1991).

8. I. Lisle, Equivalence transformations for classes of Differential Equations (University of British Columbia, Ph. D. Thesis ), (1992).

9. M. Torrisi and R. Tracinà, Equivalence transformations and symmetries for a heat conduction model, Int. J. Non-Linear Mechanics, 33, 473-487, (1998).

10. V. Romano and M. Torrisi, Application of Weak Equivalence Transformations to a Group Analysis of a Drift-Difffusion Model, J. Phys. A: Math Gen., 32, 7953-7963, (1999).

11. L. T. Takahashi, N. A. Maidana, W. C. Ferreira Jr., P. Pulino and H. M. Yang, Mathematical models for the Aedes aegypti dispersal dynamics: traveling waves by wing and wind, Bull. Math. Biol., 67, 509-528, (2005), an references therein.

12. M. Torrisi and R. Tracinà , Exact solutions of a reaction-difusion system for Proteus Mirabilis bacterial colonies, Nonlin. Anal.: Real World Appl., 12, 1865-1874, (2011).

13. J. D. Murray, Mathematical Biology, Springer, Berlin, (2002).

14. L. Dresner, Similarity solutions of nonlinear partial differential equations, Pitman Advanced Pub. Program, (1983).

15. A. Donato, Invariant solutions and nonlinear wave propagation, Int. J. Non-Linear Mechanics , 17, 307-314, (1987).

16. W. F. Ames and A. Donato, On the evolution of weak discontinuities in a state characterized by invariant solutions, Int. J. Non-Linear Mechanics, 26, 167-174, (1991).

17. M. Torrisi and M. V. Lalicata, Some similarity solutions for a hyper elastic half-space with finite deformations, Int. J. Non-Linear Mechanics , 26, 15-23, (1994).

18. M. V. Lalicata and M. Torrisi, Group-analysis approach for a binary reacting mixture, Int. J. Non-Linear Mechanics , 29, 279-288, (1994).

19. I. Sh. Akhatov, R. K. Gazizov and N. H. Ibragimov, Nonlocal symmetries: A heuristic approach, J. Soviet Math., 55, 1401-1450, (1991).

20. C. M. Khalique, F. M. Mahomed and B. P. Ntsime, Group classification of the generalized Emden-Fowler-type equation, Nonlin. Anal.: Real World Applic., 10, 3387-3395, (2009). 
21. N. H. Ibragimov and M. Torrisi, A simple method for group analysis and its application to a model of detonation, J. Math. Phys., 33, 3931-3937, (1992).

22. M. Torrisi and R. Tracinà, Equivalence transformations for Systems of First Order Quasilinear Partial Differential Equations, Modern Group Analysis VI: Developments in Theory, Computation and Application, (Edited by N. H. Ibragimov et F.M. Mahomed) New Age International Publishers, (1996).

23. M. Torrisi, R. Tracinà and A. Valenti, Group analysis approach for a non linear differential system arising in diffusion phenomena, J. Math. Phys. 37, 4758-4767, (1996).

24. N. H. Ibragimov and N. Säfström, The equivalence group and invariant solutions of a tumour growth model, Commun. Nonlin. Sci. Num. Simulat., 9, 61-69, (2004).

25. M. Torrisi and R. Tracinà, On a class of reaction diffusion systems: Equivalence transformations and symmetries, in Asymptotic Methods in Nonlinear Wave Phenomena, Eds.: T. Ruggeri, M. Sammartino, 207-216, (2007), DOI: 10.1142/97898127089080018. 